\title{
Unter dem Durchschnitt: Einige Resultate und Stärken bzw. Schwä- chen unterschiedlicher Darstellungsweisen von Ländervergleichen am Beispiel IEA-Civic Education
}

\section{Fritz Oser}

In diesem Aufsatz geht es um zwei Ziele. Erstens werden die Schweizer Resultate der IEA-Studie Civic Education, eine Vergleichsstudie über 28 Länder, zusammenfassend vorgestellt. Es zeigt sich, dass in fast allen Bereichen über die fünf inhaltlichen Schwerpunkte hinweg (1. Politisches Wissen über Inhalte, 2. Politische Interpretationsfertigkeiten, 3. Konzepte von Demokratie und Bürgerschaft, 4. Einstellungen und 5. partizipative Handlungen) die Schweizer 14/15jährigen unter dem Durchschnitt liegen. Es wird angedeutet, dass Bildungs-Politiker hinsichtlich dieser "schlechten» Resultate in einen Handlungsnotstand geraten. - Damit ergibt sich aber als zweites die Frage, ob es sinnvoll ist, in dieser Studie, aber auch bei PISA, Vergleiche über die Länder hinweg bei solch verschiedenen Dimensionen überhaupt vorzunehmen. Diese zweite Fragestellung wird kritisch unter dem Gesichtspunkt des Begriffs der "Leistungsolympiade» gewürdigt.

\section{Fragen an geistige olympiaden}

Zur Zeit erscheinen die ersten Berichte der Vergleichsstudie zur politischen Bildung (Civic Education), die die Internationale Association for the Evaluation of Educational Achievement (IEA) in verschiedenen Ländern durchgeführt hat. Für die Schweiz lag die Verantwortung bei einer Forschungsgruppe der deutschsprachigen Abteilung des Departements für Erziehungswissenschaften der Universität Fribourg. Der Begriff Civic Education könnte auch mit Staatskundeunterricht oder mit Bürgerkunde oder mit Sozialem Unterricht oder mit Ähnlichem übersetzt werden; stets würde etwas anderes mit dem Gleichen gemeint oder zumindest würde das Gleiche mit je anderem Akzent verstanden.

Wir fragen in diesem Aufsatz, welchen Aussagewert diese Vergleichsstudie hat, ob im Bereich der Staatsbürgerschaft (Citoyenität, Civics u.ä.) überhaupt Leistungsvergleiche sinnvoll sind. Die Frage ist in einem einzelnen Aufsatz nicht zu beantworten, aber sie kann im Anschluss an die vorliegenden Überlegungen präziser gestellt werden. 
In der Aufsehen erregenden Neuerscheinung «PISA 2000. Basiskompetenzen von Schülerinnen und Schülern im internationalen Vergleich» wird einleitend folgende interessante Bemerkung gemacht:

Man kann nicht nachdrücklich genug betonen, dass PISA keineswegs beabsichtigt, den Horizont moderner Allgemeinbildung zu vermessen, oder auch nur die Umrisse eines internationalen Kerncurriculums nachzuzeichnen. Es ist gerade die Stärke von PISA, sich solchen Allmachtsfantasien zu verweigern und sich stattdessen mit der Lesekompetenz und mathematischen Modellierungsfähigkeit zu konzentrieren, die nicht die einzigen, aber wichtige Voraussetzungen für die [...] Generalisierung universeller Prämissen für die Teilhabe an Kommunikation und damit auch für Lernfähigkeit darstellen (Baumert u.a. 2001, S. 21).

Gilt das Gleiche für den Bereich der politischen Bildung, oder geht es bei dieser Untersuchung eben doch um mehr, nämlich um eine Art Allgemeinbildung, die nicht die allgemeine Kommunikationsfähigkeit, wohl aber die verantwortliche Partizipation am politischen Geschehen des eigenen Landes und eben die allgemeine politische Bildung im Visier hat. Wenn man zeigen kann, a) welches inhaltliche Wissen, b) welche Fertigkeiten für die Interpretation von «zivilem» und politischem Inhalt, c) welche Konzepte und Vorstellungen des Politischen, d) welche Einstellungen (attitudes) zum Beispiel hinsichtlich des Vertrauens in die Regierung und e) welche gegenwärtigen und in der Zukunft erwarteten partizipatorischen Handlungen vorhanden sind oder sichtbar werden, so kann man erzieherisch mehr anvisieren als bloss Grundkompetenzen. Es sind entscheidende Bildungsvoraussetzungen, die mit der Erhaltung demokratischer Staatssysteme bzw. mit den Entwicklungsmerkmalen der westlichen Zivilisation in Zusammenhang stehen. Die fünf erwähnten Bereiche sind denn auch der Kern der erwähnten Untersuchung mit dem Titel «Citizenship and Education in Twenty-eight Countries. Civic Knowledge and Engagement at Age Fourteen", die von TorneyPurta u.a. (2001) herausgegeben worden ist, und an dem unsere Forschungsgruppe die Daten für die Schweiz zu verantworten hat ${ }^{1}$. Diese fünf Bereiche werden ergänzt durch drei inhaltliche Gruppen, nämlich I a) Demokratie, b) Bürgerschaft (Citizenship); II a) nationale Identität, b) internationale Beziehungen; und III a) soziale Kohäsion, b) Diversität.

An unserer Studie haben, wie im Titel angedeutet, 28 Länder teilgenommen, nämlich Australien, Belgien (französisch), Bulgarien, Chile, Kolumbien, Zypern, Tschechische Republik, Dänemark, England, Estland, Finnland, Deutschland, Griechenland, Hongkong (SAR), Ungarn, Italien, Lettland, Litauen, Norwegen, Polen, Portugal, Rumänien, Russische Föderation, Slowakische Republik, Slowenien, Schweden, Schweiz und USA (Reihenfolge alphabetisch nach der englischen Schreibweise). Die jeweiligen 14/15jährigen Schüler und Schülerinnen wurden in Klumpenstichproben (Schulen) ausgewählt. Für die Schweiz beteiligten sich 3104 Schüler und Schülerinnen aus zufällig über die Kanone gezogenen 157 Schulen. 
Wenn nun die Resultate der Länder in Bezug auf die oben aufgeführten Dimensionen und Inhalte verglichen werden, so kann einerseits festgestellt werden, dass eine Gruppe von Ländern im jeweiligen Bereich eben besser abschneidet oder aber verglichen mit dem Mittelwert über alle andern Länder hinweg schlechter dasteht. Es handelt sich um eine Art Olympiade, die nichts aussagt über das Mögliche, sondern nur vergleichend über das Bestehende. Man könnte sich ja vorstellen, dass alle Länder im Bereich der "Civic Education» schlecht abschneiden und dass somit der Vergleichswert nicht sonderlich gross ist, weil er sich stets in einem unteren Bereich befindet. Wenn alle schlecht wären, dann könnte man nichts über die Qualität aussagen. Damit stehen wir im Zentrum der Problematik solcher Vergleiche. Sie beinhalten nur vergleichend Informationen über Qualität, auch nur deskriptiv etwas über vorgestellte Erreichung von Zielen, kaum etwas über mögliche Optimierung von Einstellungen, sie sind also nur wertvoll über die Relativität einer Leistung im Vergleich zur Leistung anderer Länder und ihren erzieherischen oder unterrichtlichen Gepflogenheiten. ${ }^{2}$ Auch das hat Vorteile, vor allem aber Nachteile. Der Nachteil ist, dass die Güte der Leistung oder der Einstellung immer relativ zu andern erfasst wird. Der Vorteil ist, dass die Sache der Qualität auch ganz anders angegangen werden kann, nämlich indem nicht im Voraus eine hypothetische - und hier nicht definierte normative - «Qualität» vorausgesetzt wird, sondern der Vergleich eben dazu genützt wird festzustellen, was in andern Ländern, mit andern Voraussetzungen und andern Ansätzen tatsächlich erreicht wird, d.h. eben möglich ist. In diesem Sinne beinhalten die Daten, sowohl von PISA wie in Civics Education, viel über "Qualität», aber diese wird durch Kriterien definiert, welche in die empirische Messung der Wirkungen einfliessen, die sich aus Schulung sowohl als aus andern Einflüssen zu einem bestimmten Zeitpunkt des Lebenslaufs ergeben. Diese «Qualität» bleibt eine relative, auch wenn sie eher für die "versagenden» Länder interessant erscheint.

Die erwähnte Olympiade hat ihre eigenen stichprobentheoretischen Probleme, ihre eigenen kulturellen Missverständnisse dessen, was abgefragt wird, ihre eigenen Erklärungsweisen für Umgang mit den Gründen für Unterschiede und ihre eigenen bildungspolitischen Reaktionen. Nichtsdestoweniger ist sie geeignet, ein Bewusstsein des Möglichen zu schaffen, gerade weil auch forschungstechnisch all diesen Studien ein hoher Standard zugebilligt werden muss. Freilich ist dies nur möglich, wenn die Grundsätze der Leistung, die unumgänglicher Teil schulischen Lernens sind, eine neue Reflexion und eine neue Akzeptanz erfahren. Man muss sich fragen, wie vielfältig Leistung sein kann, vor allem wenn sie sich auf die Gestaltung eines politischen Nahraums bezieht, den Forscher schwerlich kennen können. Noch mehr aber muss gefragt werden, was junge Personen, die auf unsere Fragen antworten, meinen; geht es ihnen um den von Beck (1997) beschriebenen Individualismus mit der entsprechenden Angst vor der Freiheit, die schon immer in Grenzen praktiziert wird und die nach vorn blicken lässt, oder um ein traditionelles Wertesystem, das die Untersucher mit 
ihren Fragen abzurufen gedenken? Das Erhebungsparadox kann hier vermutlich nicht ganz gelöst werden: auf der einen Seite muss mit der Tradition der Vorstellungen gearbeitet werden; Auf der anderen Seite fliesst der neue Geist immer schon mit in die alten Fragen ein.

\section{Der Begriff “Citizenship and Education»}

Für den in diesem Titel aufgeführten Begriff "Citizenship» gibt es keine adäquate deutsche Übersetzung. In ihm drückt sich nicht bloss Erziehung zur Demokratie oder Staatskunde aus; mit diesem Begriff ist mehr gemeint. In einer sprachlich-erzieherischen Analyse sagt Berkowitz:

There are two common ways to understand civics education: education about civics and education for citizenship. They are not unrelated, but neither are they redundant. The former concerns the traditional didactic presentation of information about one's society; e.g. the particular form of government, the roles and obligations of citizens, etc. The latter is more a matter of nurturing the development of the individual, in this case the development of those attributes necessary to function as an effective citizen of the society; e.g. the ability to understand and evaluate relevant issues, the willingness to advocate for the society and the civic process, etc.» (2000, S. 898).

Und Reichenbach (1999, S. 391) bemerkt in einer Fussnote, dass Citoyenität auf gesellige und öffentliche Sozialverbindungen hinweise, also mehr meine, als was mit dem Begriff der Bürgerschaft normalerweise anklingt. Es ist das engagierte Verhalten, das das gesellige Benehmen impliziert und in Ökonomie und Öffentlichkeit, aber nicht im politischen Staat, den Ort der Wirkung findet. Sie ist aber auch nicht die Privatsphäre, obwohl Fragen der Realisierung des Politischen oder des Demokratischen dort eine Rolle spielen können (vgl. Taylor 2002, S. 84). In der Analyse dessen, was Öffentlichkeit ist, stellt Taylor deshalb gerade diese dem organisierten Staat entgegen; sie ist das nicht organisierte, das aber wichtige Funktionen für das Gestalten des staatlichen Willens ausübt. Sie ist das Forum der Freiheit, das die Plattform für Diskurse über den Staat, die nicht erzwungen werden können, erst entwickelt.

Es wird schwierig sein, in unserer Untersuchung, diesen Begriff adäquat zu gebrauchen. In der von Reichenbach durchgeführten Vorstudie zur Civic Education in Switzerland, in der Experten aus Unterricht, Ausbildung und Politik (qualitativ) befragt worden sind, kommt denn auch eine gewisse Hilflosigkeit angesichts der Vielfalt dieses Begriffs deutlich zum Ausdruck (vgl. Reichenbach, 1998).

Für unsere Studie haben wir einen pragmatischen Zugriff auf Citizenship angezeigt, denn im Grunde möchten wir uns an die Operationalisierungen, wie sie in der Untersuchung vorgenommen worden sind, halten. Diese waren übrigens in ihrer Genese hoch interessant, denn alle Vorschläge für Items wurden von den National Coordinators in einem engagierten Diskurs abgestimmt. Viele Items 
wurden herausgenommen, weil der entsprechende Inhalt in einzelnen Ländern formal einfach nicht existierte. Es konnte, um nur ein Beispiel zu erwähnen, in andern Ländern als der Schweiz kaum von Abstimmung gesprochen werden, denn viele Länder haben wohl Wahlen, aber keine Abstimmungen.

\section{Resultate der erwähnten Untersuchung}

Die Darstellung einiger Befunde aus der Studie soll hier nur wenig Raum einnehmen, weil der Wunsch, nur eine Übersicht über das Wesentliche zu erhalten, ansonsten beeinträchtigt würde und weil wir an anderer Stelle über die Gesamtstudie berichten werden. Freilich soll trotz dieser Kürze die Stellung der Schweizer 14/15 -Jährigen im Vergleich zu den Jugendlichen in den andern 27 Ländern herausgehoben werden, damit anschliessend nochmals nach dem Sinn und Zweck solcher Vergleiche besser nachgefragt werden kann.

\section{Wissen und Fertigkeiten (Skills)}

Dieser Teiltest bestand aus 25 Frage-Items zum politischen Wissen des Genres, welche von den vorgegebenen Antworten etwa am meisten dafür spreche, dass eine Regierung als "undemokratisch» bezeichnet werden könne, a) wenn die Menschen in diesem Land daran gehindert werden, die Regierung zu kritisieren; b) wenn die politischen Parteien sich gegenseitig oft kritisieren; c) wenn die Leute hohe Steuern zahlen müssen; d) wenn jeder Bürger das Recht auf eine Arbeit hat. Die so genannten 13 Skillfragen haben mit der Interpretation von politischen Situationen oder Texten zu tun. Da wird z.B. gezeigt, wie jemand aus einem Geschichtsbuch etwas ausradiert, und es steht die Frage dabei, was die Botschaft dieser Zeichnung sei, nämlich Geschichtsbücher für die Schule würden a) manchmal geändert, damit problematische Ereignisse aus der Vergangenheit nicht erwähnt werden müssten; b) manchmal kürzer seien als Geschichtsbücher für Erwachsene; c) voll von Informationen seien, die uninteressant wären; d) sollten mit Computer und nicht mit einem Stilo geschrieben sein. Die obige Wissensfrage wurden im Durchschnitt von 53 \% (Streuung zwischen 36 und $73 \%$ ) richtig beantwortet. Sie ist verglichen mit dem Durchschnitt aller gelösten Items (64\%) relativ schwierig. Die Interpretationsfrage wurde von 57\% aller Schüler und Schülerinnen richtig gelöst (Streuung 26 bis 79\%). Der Schwierigkeitsgrad der Items wurde hierarchisiert und in einen Gesamtzusammenhang eingebaut.

Gesamthaft gesehen sind die beiden Skalen über die Länder hinweg valide, reliabel und bedeutsam. Die Unterschiede zwischen den Ländern sind klein; 25 von ihnen differieren weniger als ein halbe Standardabweichung. Unterschiede zwischen Knaben und Mädchen gibt es nur bei einzelnen Items; sie sind ähnlich über die unterschiedlichen Länder hinweg. Schüler und Schülerinnen, die angeben, zu Hause Lesequellen zu haben, schneiden besser ab als solche, die dies 
nicht angeben. Über 75\% der Befragten können Fragen beantworten, die mit der Natur der Gesetze und der politischen Rechte zu tun haben. Hingegen können nur wenige mit jenen Fragen umgehen, die mit komplexeren Gebieten zu tun haben, wie etwa die Wahl der Kandidaten politischer Parteien auf der Basis der politischen Programme oder Reform politischer Institutionen u.ä.. Die Schweiz liegt im Wissenstest im Mittelteil der 28 Nationen, das heisst, die Schweizer Werte, obwohl nur an neunzehnter Stelle von 28 Nationen liegend, weichen nicht signifikant vom Mittelwert über diese 28 Nationen ab. Hohe Werte erreichen Polen, Finnland, Zypern und Griechenland, die tiefsten Werte erreichen Kolumbien, Chile, Litauen und Rumänien. Der Unterschied innerhalb unseres Landes ist frappant. Die italienisch sprechenden Schüler und Schülerinnen liegen sehr viel höher als die französisch sprechenden und diese wiederum höher als die deutsch sprechenden. Würde man die deutschsprachigen Schüler und Schülerinnen allein betrachten, so wären sie am fünftletzten Platz aller 28 Nationen anzusiedeln!

Bei den Interpretationsfähigkeiten (Skills) ist die Streuung über die Länder grösser; die Platzierung in der Rangfolge ist ähnlich. Die Schweiz liegt aber signifikant unter dem Mittelwert der Nationen. Hier schneiden vor allem die italienisch sprechenden Jugendlichen sehr gut ab. Die deutsch und französisch sprechenden liegen unterhalb der Mitte. Man kann sich also fragen, warum die Tessiner Jugendlichen soviel besser als die deutschsprachigen oder welschen 14/15 -Jährigen in den Wissens- und Interpretationsfragen antworten.

\section{Konzepte von Demokratie, Bürgerschaft (Citoyenität) und Regierung}

Was sich junge Menschen über Demokratie denken, ist nicht gleichgültig, denn Veränderungen entstehen später stets aus den Erwartungen, die mit Konzepten zusammenhängen. Auch für den Unterricht sind Konzepte von grosser Bedeutung, stellen sie doch einen Teil des Vorwissens von Schülern und Schülerinnen dar, auf dem aufgebaut wird. Die Hauptfrage war hier, ob junge Menschen sehen, was die Demokratie fördern oder behindern kann. Ein Itembeispiel ist, ob es für Demokratie «sehr gut», "gut», «weniger gut» oder «gar nicht gut» sei, wenn es in ihr Vereinigungen gibt, denen sich Menschen anschliessen möchten. - Ein Resultat ist, dass 14/15 -Jährige glauben, dass freie Wahlen, die aus der Verfügbarkeit vieler Organisationen, Zusammenschlüsse und Gruppen der Bürger und Bürgerinnen hervorgehen, die Demokratie stärken, dass es hingegen schlecht sei, wenn reiche Leute die Regierung beeinflussen können oder wenn die Politiker die Gerichte beeinflussen und den Menschen verbieten, die Regierung zu kritisieren. Die Autoren des Grundberichtes haben jene Items gruppiert, die hohe, mittlere und kleine Übereinstimmung zwischen den Nationen erfahren. Interessant für die Schweiz ist, dass etwa ein Item, wie, dass es schlecht sei für die Demokratie, wenn reiche Leute auf die Regierung Einfluss haben, als für die Demokratie allgemein schlechter eingeschätzt wird als bei anderen Staaten; das 
Recht, die eigene Meinung sagen zu können, wird hingegen, positiver eingeschätzt als beim Durchschnitt der anderen Staaten. Dies sind nur zwei Beispiele.

Das Konzept der Bürgerschaft (Staatsbürgerschaft) oder Citizenship (Citoyenität) wird auf zweierlei Weise konzeptionell vorgestellt, nämlich als konventionelle und als sozial-engagagement-orientierte. Das erstere hat zu tun mit dem Wahlverhalten, der Parteizugehörigkeit, dem Geschichtsbewusstsein, den Erkundigungen über das Radio, das Fernsehen oder die Zeitung. Es beinhaltet auch Respekt für Politiker und politische Diskussionen. Hier schneidet die Schweiz knapp, aber signifikant unter dem Mittelwert der verschiedenen anderen Länder ab. Bei der sozial-engagement-orientierten Bürgerschaft, wo es um Demonstrationsrecht, Beteiligung an Hilfsaktionen, Menschenrechte und Umweltschutz geht, liegen die Schweizer Schülerinnen und Schüler im unteren Drittel der Nationen. Gesamthaft gesehen haben 14/15 -Jährige eine Vorstellung von Bürgerschaft, die sehr viel mit der Einhaltung von Gesetzen zu tun hat. Wählen ist wichtig, nicht aber bei einer politischen Partei zu sein oder das Engagement in politischen Diskussionen. Das spielt fast keine Rolle.

Auch beim Konzept «Regierung» sind zweierlei Skalen eingesetzt worden, nämlich eine, die sich auf gesellschaftsbezogene und eine die sich auf wirtschaftsbezogene Verantwortung der Regierung bezieht. Bei beiden liegen die Einschätzungen der Schweizer Schüler und Schülerinnen im untersten Fünftel der Nationen, dies obwohl gesagt werden kann, dass unsere 14/15 -Jährigen schon sehr solide Vorstellungen bzw. Konzepte der sozialen und ökonomischen Verantwortung der Regierung haben. Freilich wird das «Sich-Einsetzen für Erziehung» höher gewichtet als «Einkommensunterschiede auszugleichen» oder «Preise zu kontrollieren.»

\section{Vertrauen in staatliche Institutionen und Medien, und die Einstellung zur Nation}

Ob junge Menschen Vertrauen in staatliche Institutionen und in die Presse haben, steht nicht zuletzt in Zusammenhang mit dem Konstrukt der so genannten Politikverdrossenheit. Man kann sich zweierlei vorstellen, nämlich, dass eine Person politisch unbeteiligt bleibt, weil sie Vertrauen hat und «die da oben» schon alles recht machen, oder weil man kein Vertrauen hat und dadurch auch eine gewisse Protesthaltung zum Ausdruck bringen will. Die Fragen lauten also, wie sehr der Schweizer Regierung, dem Stadt- oder Gemeinderat, den Gerichten, der Polizei, den politischen Parteien der Kantonsregierung oder bezüglich den Medien dem Radio, dem Fernsehen, den Zeitungen Vertrauen entgegen geracht. Flanagan, die (2000) ebenfalls eine Studie zu politischem Wissen, zur Einstellung und zum Verhalten von über 5000 12- bis 19 -Jährigen in sieben Ländern durchgeführt hat, meint, dass Vertrauen in die Regierung und die politischen Institutionen ein zentrales Element des Politischen schlechthin sei. Beck (1997) behauptet aber, dass die Kinder der Freiheit, indem sie nur Vertrauen in das Unmittelbare haben, hochpolitisch handeln täten. 
Welches sind die Resultate? Die 14/15 -Jährigen Schweizer und Schweizerinnen liegen in Bezug auf das Vertauen in die staatlichen Institutionen an drittoberster Stelle (nach Dänemark und Norwegen); nach ihnen folgen Zypern und Griechenland. Am schlechtesten schneiden Slowenien, Bulgarien, Russland und Litauen ab. Zugleich aber vertrauen die Schweizer Jugendlichen weniger als diejenigen anderen Ländern in die politischen als in die andern Institutionen. Hohes Vertrauen weist auf die Stabilität der Regierungsinstitutionen bzw. der Demokratie als Ganzes hin. Geschlechtsspezifische Unterschiede sind diesbezüglich für die Schweiz nicht vorhanden. - Was die Medien anbelangt, sind es etwa $52 \%$, die Vertrauen haben; beim Fernsehen- und dem Radio weit unter dem Durchschnitt, bei den Zeitungen, höher bzw. beim Durchschnitt. - Dieser Befund muss in Zusammenhang mit dem Vertrauen in die Institutionen gesehen werden. Zweifelsohne kann man annehmen, dass die öffentlichen Medien als viel beeinflussbarer als die Regierungsinstanzen gesehen werden bzw. dass sie eher als Forum der Auseinandersetzung mit all den Wertungen und Grenzen des Wissens behaftet eingeschätzt werden als die offiziellen staatlichen Instanzen. Der Zusammenhang zwischen dem Vertrauen in die Regierungsinstitutionen und einem gewissen mittleren Misstrauen gegenüber der Presse und dem Radio/Fernsehen ist also in der Schweiz ideal. Diese Situation ist für die Schweizerische Demokratie vermutlich konstituent.

Wie gross ist die Identifikation unserer Jugendlichen mit der Nation? Die Fragen waren so strukturiert, dass Zustimmung gegeben werden musste zu Aussagen wie, dass einem die Schweizerfahne wichtig sei, dass man die Schweiz liebe, dass man stolz sein solle auf das, was erreicht worden sei oder (umgekehrt gepolt) ob man dauernd in einem andern Land leben, also die Schweiz verlassen möchte. Im Vergleich zu den 28 Ländern steht die Schweiz an viertletzter Stelle. Hinter ihr sind noch Deutschland, Hongkong und Belgien. $\mathrm{Zu}$ oberst finden sich Griechenland, Zypern, Chile und Polen. Einerseits könnte dieses Resultat ein Hinweis darauf sein, dass Vertrauen in die politischen Instanzen nicht gleichzeitig eine stärkere nationale Identität bewirkt. Andererseits stimmen nur 58\% der Schweizer Jugendlichen (im Vergleich zu 82\% im Durchschnitt aller befragten Länder) zu, dass ihnen die Fahne wichtig sei. 78\% lieben ihr Land, 84\% glauben, dass man stolz sein soll darauf, was erreicht worden ist und $32 \%$ können sich vorstellen, irgendwo anders auf Dauer leben zu können (im Vergleich zu 23\% der andern beteiligten Nationen). Das sind interessante Resultate, die auf eine besondere Art von aufgeklärtem Umgang mit nationaler Identität hinweisen.

Bei der Diskussion dieser Resultate ist allerdings zu beachten, dass zwei wichtige Dimensionen fehlen, nämlich die Identifikation mit den anderen Formen des föderalistischen Systems (z.B. mit dem Kanton) und dass der Ausländeranteil an dieser Einschätzung in einem weiteren Schritt unabhängig vom Schweizer Anteil analysiert werden muss, dies weil Ausländer oft eine starke Identität mit ihrem Heimatland mitbringen. 


\section{Chancengleichheit von Immigranten und Frauen}

Ein Teil der hier vorliegenden Untersuchung handelt von aktuellen Problemen in der Gesellschaft, so von den Einstellungen bezüglich der Rechte der Immigranten und der Chancengleichheit der Frauen. Die Items für die erste Gruppe betreffen z.B. die Möglichkeit, die eigene Sprache weiter zu sprechen, den Kindern die gleichen Bildungschancen zu gewähren, bei längerem Aufenthalt wählen zu können, ja sogar ob sie gleiche Rechte wie jeder andere haben sollten.

Gesamthaft gesehen liegen die Zustimmungen zu allen Bereichen über alle teilnehmenden Länder hinweg zwischen 77 und 90\%. Nur wenige lehnen das Recht auf die eigene Sprache ab und nur 3\% wollen die gleichen Bildungschancen nicht verwirklicht sehen. 6\% sind nicht für Wahlberechtigung und nur 5\% wollen überhaupt keine Rechte verwirklicht sehen. In diesem relativ positiven Zustimmungsgeflecht steht aber die Schweiz an zweitunterster Stelle (hochsignifkant tiefer vom Mittelwert der 28 Länder). Nach ihr liegt nur noch Deutschland, vor ihr Slowenien, Lettland, Ungarn; zuoberst liegen Zypern, Kolumbien, Schweden, Griechenland und Polen, in der Mitte liegen die USA, Rumänien, Australien und Belgien. Die Ausdifferenzierung nach dem Anteil der Ausländer und Ausländerinnen scheint keinen signifikanten Einfluss auf das Gesamtergebnis zu haben.

Bei den Rechten der Frauen geht es um Fragen wie Kandidatur für öffentliche Ämter und Regierungen, um gleiche Rechte wie Männer, auch um Formulierungen wie "Frauen sollten in der Politik nichts zu suchen haben», um Notsituationen wie Arbeitslosigkeit und ob da Männer mehr Chancen haben sollten als Frauen, um gleiche Bezahlung für gleiche Arbeit und um geschlechtsspezifische politische Führungseigenschaften. Die Gesamtresultate sind hier recht positiv. In der Gesamtstichprobe sprechen 89\% aller Schüler und Schülerinnen den Frauen das Recht zu, sich an der Regierung zu beteiligen oder ein Amt innezuhaben. 90\% sind für gleiche Rechte für Frauen. 75\% lehnen ab, dass Männer bei Arbeitslosigkeit mehr Rechte als Frauen haben sollten. 91\% sind für gleiche Bezahlung, und $72 \%$ glauben nicht, dass Frauen weniger geeignet sind zur politischen Führung. Bezüglich dieser Rechte schneiden die Schweizer Schüler und Schülerinnen an siebt oberster Stelle ab; es werden also nur von einer Minderheit Diskriminierungen aufrechterhalten. An oberster Stelle liegen Dänemark, Norwegen Australien und England; vor der Schweiz liegen Deutschland und dahinter die USA; in der Mitte liegen Polen Portugal, Griechenland und Italien; am Schluss liegen Russland, Lettland Rumänien und Bulgarien. Ein geschlechtspezifische Teilanalyse zeigt nun allerdings, dass die Mädchen stärker bei beiden, den Migranten- und besonders stark bei den Frauenrechten, Unterstützung zum Ausdruck bringen, und dies nicht nur in der Schweiz, sondern in sämtlichen 28 Ländern. Es liegt hier der stärkste geschlechtspezifische Effekt der gesamten Studie vor. 


\section{Civic Engagement und politische Aktivitäten}

Eine der entscheidenden Fragen der gesamten Untersuchung bezieht sich auf das Engagement der Jungen, also darauf, ob sich Schülerinnen und Schüler an der Gesellschaft jetzt und später auch politisch engagieren würden. Diese Frage hängt zusammen mit der Teilnahme an politischen Entscheidungen einerseits und mit den politischen Interessen andererseits. In einer spontanen Hypothese kann man leicht behaupten, dass das Bestehen eines Staatsgebildes und die Verteidigung der Freiheitsrechte gegenüber jeglicher Art von Eingriffen mit dem politischen Interesse und dem potentiellen Engagement zusammenhängt. Pädagogische Forderungen und Ziele richten sich besonders darauf aus, ob und wie dieses Interesse stimuliert werden kann.

Die Fragen zum Interesse wurden durch ein direkt gestelltes Item «Ich bin an der Politik interessiert», und durch weitere indirekte Interessensitems (über die Häufigkeiten, mit denen die Schüler und Schülerinnen Nachrichten hören oder sehen, Zeitungen nach politischen Ereignissen absuchen etc.) erfragt. Das Resultat hat zwei Seiten: Erstens rapportieren die Jugendlichen allgemein moderates Interesse an Politik. Nur in Kolumbien, Russland, Zypern und in der Slowakei zeigen mehr als 50\% starkes Interesse an politischen Fragen. Zweitens befindet sich die Schweiz mit 33\% der interessierten Schüler und Schülerinnen im letzten Fünftel der Nationen.

Eine Reihe weiterer Fragen beschäftigten sich mit Teilaspekten politischer Aktivität. Wiederum schneiden die Schweizer Jugendlichen sehr moderat ab. 92.1\% haben «nie in einer Schülervertetung» mitgemacht; 96.1\% waren nie in einer Jugendorganisation, einer Jugendpartei oder Gewerkschaft tätig; 82\% haben nie zur Redaktion einer Schülerzeitung beigetragen; 90\% waren nie in einer Umweltorganisation; 99.15\% haben nie in einem UNO- oder einem UNESCO- Klub mitgearbeitet; $85.3 \%$ haben nie an einem Schüleraustauschprogramm teilgenommen; $97.3 \%$ waren nie in einer Menschenrechtsorganisation tätig; $88.6 \%$ haben nie in einer Gruppe, die freiwillig Dienste für die Gesellschaft leistet, gewirkt, und 73.1\% haben nie in einer Gruppe, die Spenden für soziale Zwecke sammelt, mitgearbeitet, so stellt dies Maiello (vgl. Oser et al. in Vorbereitung) in unserem Rapport fest.

Man kann sagen, dass die politischen Aktivitäten von Schweizer Jugendlichen im Rahmen von politischen oder teilpolitischen Organisationen wenig ausgeprägt sind. Auch die zukünftige Partizipationsabsichten scheinen sich auf das Wählen und die Informationssuche betreffs Kandidaten der politischen Parteien zu beschränken. Innerhalb der Schweiz scheint also das politische Interesse mittelmässig ausgeprägt und im internationalen Vergleich unterdurchschnittlich zu sein. Interessant sind auch die Geschlechtsunterschiede: Mit den Ausnahmen von Schweden und den USA haben Mädchen weniger hohe Erwartungen als Knaben betreffs politischer Partizipation. 


\section{Partzipationsmöglichkeiten in Schule und Jugendorganisa-} tionen

Der Einfluss der schulischen Partizipation auf das spätere politische Verhalten ist eine der noch unbekannten Grössen in den Attributionen politischer Erziehungsabsichten. Oser und Biedermann (2001) konnten zeigen, dass soziales Engagement kaum mit gegenwärtigem politischem Engagement korreliert. Man nimmt zwar an, dass die Schule als System so etwas wie eine res publica sein könnte und dass die hier "geübten» vorpolitischen Verhaltensweisen gleichsam eine Wirkung auf die politische Sensibilität junger Menschen für morgen hätten.

Eine erste Skala bezieht sich deshalb auf die geglaubten Veränderungen durch persönliche Partizipation. Sie stellt so etwas wie eine politische Selbstwirksamkeitsskala dar mit Fragen, ob es besser werde, wenn Schülervertreter gewählt würden, ob es Veränderungen gäbe, wenn Schüler zusammenarbeiten würden, ob man helfen könnte, Probleme zu lösen, wenn Schülergruppen organisiert ihre Meinung äusserten, ob Zusammenarbeit einen Einfluss auf das, was an der Schule passiert hätte. Bezüglich dieser Selbstwirksamkeit stehen die Schweizer Schüler und Schülerinnen unter dem Durchschnitt im Nationenvergleich an drittletzter Stelle. Nur Ungarns und Deutschlands Jugendliche haben noch weniger Vertrauen in die Wirksamkeit ihrer eigenen schulischen Partizipation. Soziale Kompetenzen, die man als Voraussetzung für politisches Denken bezeichnen könnte, sind hingegen positiv ausgeprägt. Items wie «in der Schule habe ich gelernt, mit andern zusammenzuarbeiten, Menschen mit unterschiedlichen Ideen zu verstehen, zur Lösung von Problemen in der Gesellschaft beizutragen», schlagen sehr positiv aus (74 bis $92 \%$ ja). In der Schule habe ich gelernt, nationalbewusst und ein guter Staatsbürger zu sein, an den Wahlen teilzunehmen, die Umwelt zu schützen, schlagen nur mittelmässig aus (44 bis 64\%, wobei das Nationenmittel von 55 bis $79 \%$ steht).

Freie Meinung im Klassenzimmer ist eine andere Grösse, die man als Voraussetzung für politische Bildung bezeichnen könnte. Die Items beinhalten Fragen zum Widerspruch, zur eigenen Meinung, zum Respekt vor anderer Meinung, zum Mut, eine abweichende Meinung zu formulieren, zur Ermutigung von Diskussion unterschiedlicher politischer Ansichten, zur Offenheit für gegensätzliche Ansichten im Unterricht. Als Resultat ist festzuhalten, dass sich die Schweiz hier im vordersten Viertel des internationalen Ländervergleichs befindet. Zuoberst finden wir Kolumbien, Norwegen, die USA und Griechenland, zuunterst Bulgarien, französisch Belgien, Slowenien und Ungarn. In 23 der 28 untersuchten Länder geben Mädchen an, deutlich häufiger Möglichkeiten der freien Meinungsäusserung als ihre männlichen Kollegen haben zu können.

Als Gesamtresultat stellen wir hier einen interessanten Zwiespalt fest. Partizipativen Prozessen wird in der Schweiz grosser Wert beigemessen; mit anderen Jugendlichen zusammenzuarbeiten, Menschen zu verstehen und unterschiedliche Ideen zu haben, wird als vorhanden und überzufällig gegeben dargestellt. Hingegen schneidet die Schweiz gegenüber des geäusserten Vertrauens hinsichtlich 
der Wirksamkeit schulischer Partizipationsprozesse eher schlecht ab. Das Engagement an quasipolitischen und vorpolitischen Institutionen ist sehr tief.

Soweit eine Zusammenfassung der Schülerdaten.

\section{Bei fehlender Goldmedaille, die Suche nach den Schuldigen}

Im Zusammenhang mit den hier vorgestellten Daten sei nochmals die kritische Frage angebracht, was denn solche Vergleichsstudien, die wir als geistige Olympiaden bezeichnet haben, überhaupt leisten können. Es gibt eine ganze Reihe von Ansichten und Reaktionen; zwei davon sind sehr problematisch. Vom Glauben beherrscht, dass dies schliesslich zu relativieren sei und dass man noch zu wenig über die Gründe des Versagens wisse, ist vermutlich die Ansicht der Erziehungsdirektorenkonferenz ziemlich fragwürdig. Sie will nämlich erst einmal zuwarten, bis nähere Ergebnisse (hier natürlich nur in Bezug auf PISA) auf dem Tisch liegen. Anstatt einen Diskurs darüber zu führen, ob man mehr Leistung will und dann mehr Leistung zu erwarten (die Professionellen sollen selber wissen, wie dies zu erreichen wäre und könnten Ansprüche stellen), wird das Ganze aufgeschoben bzw. zu einem Nulltarif aufgerechnet, obwohl die Schweiz gesamteuropäisch verglichen relativ wenig für Erziehung und Bildung ausgibt. Für die politische Bildung würde das bedeuten, dass man ebenfalls zuwartet, bis andere Länder Entscheidungen von tiefgreifender Wirkung fällen, so etwa ein Spiralcurriculum für Geschichte und Politikunterricht entwerfen, das äusserst attraktiv ist, oder neue Entscheidungen treffen, damit die Schulen als kleine «Praxis der Freiheit» (Arendt 1996) für bestimmte Bereiche parlamentarische Prozesse für alle Beteiligten ermöglichen und so eine politische Schulkultur aufbauen könnten (Just Communities).

Die zweite Haltung ist diejenige der Lehrpersonen, die offiziell über den Lehrervereinspräsidenten oder über die Inspektorenvereinigung ausrufen lässt, dass die Lehrpersonen (hier auch für PISA) keine Verantwortung übernehmen können. «Lehrerpersonen tragen keine Schuld» lauten die ganzseitigen Headlines der Freiburger Nachrichten vom 19. Januar 2002. - Ich meine, dass Professionelle auf alle Fälle einen Teil der Verantwortung übernehmen sollten, wenn Schüler und Schülerinnen wenig lesen können oder wenig politisches Wissen haben. Ein Dachdecker übernimmt für seine Arbeit Verantwortung, auch ein Bierbrauer tut dies, auch ein Arzt etc. Lehrpersonen bemühen sich um das Lernen der Kinder, und sie trifft eine Verantwortung, auch wenn wir alle wissen, dass die Zeiten schwer geworden sind und der Klassenraum ein emergency room mit unendlich vielen feinen Ansprüchen geworden ist. Nicht dass mit Fingern auf den Lehrerstand gezeigt wird, aber der Lehrerstand selber muss diese Resultate vorerst als Evaluationsresultate mit selbstregulativer Wirkung zur Kenntnis nehmen. Lehrpersonen erhalten gerade dann, wenn sie sich verantwortlich fühlen, einen Bo- 
nus gesellschaftlicher Akzeptanz. Der Unterricht, auch wenn «school matters» als Fazit gegen den Unterricht als alleinige Wirkungsquelle angenommen wird, muss eine neue Kultur der Anstrengung entwickeln. Für Civic Education bedeutet dies, dass die Lehrerschaft eine neue Verantwortung übernimmt für die politische Identitätsentwicklung der Adoleszenten, dies ohne die Abwehr durch den Diskurs der Überlastung und ohne den fatalen Rückzug aus dem Widerstreit, der solchen Prozessen immer zugrunde liegt.

Diese zwei Haltungen sollen aber nicht über die Frage hinweg täuschen, ob Olympiaden dieser Art etwas Zwiespältiges an sich haben. Es wird stillschweigend angenommen, dass bessere Leistung (vor allem aber Medaillen im Ländervergleich) unter allen Umständen anzustreben wäre, weil wir damit in Konkurrenz zu anderen Gesellschaften bestehen könnten. Es wird angenommen, dass hohe Anstrengung in den untersuchten Bereichen ein «Hypergut» darstellt, das auf alle Fälle und mit allen Mitteln zu erreichen sein müsste. Wie sieht dies alles im Bereich Civic Education aus? Politische «Leistung» wäre dann hohes Vertrauen in die Regierung, differenzierte politische Haltung, grosses Wissen, ausgeprägte Skills, grosses sozial-politisches Engagement, hohe Kohäsion usw. Freilich wird man den Gedanken nicht los, dass dies alles nicht unbedingt gefördert werden sollte, dass die Gesellschaft als solche diese Werte nicht unbedingt hochhalte, dass Politiker nicht unbedingt eine vorbildliche Voraussetzung für solches schaffen, aber besonders dass Schule nicht gegen die Gesellschaft erziehen könne. Kommt dazu, dass kein einheitliches Curriculum mit ansprechbaren Zielen zur politischen Bildung existiert, was eine Vernachlässigung der Erziehungsdirektorenkonferenz, nicht aber der Lehrpersonen darstellt. Die Bedingungen sind in diesem Bereich suboptimal. Das Ernstnehmen der Vergleiche müsste ja ein neues Programm in Bewegung setzen, einen Fortschritt ins Auge fassen, neue verbindliche Lehr-Lern- und Erziehungsprozesse bewirken. Wir haben vergessen, dass wir erst noch Standards im Bereich der politischen Bildung entwickeln müssen.

Dieser Zwiespalt wird doppelt bewusst, wenn man die Datenpräsentation im internationalen Report mit derjenigen unseres Schweizer Berichtes, der anfangs 2003 erscheinen wird, vergleicht. Torney-Purta et al. haben nur die Resultate des Wissenstests ranghierarchisch geordnet; sie begründen dies damit, dass man Einstellungen und Haltungen nicht hierarchisieren könne. Tab. 1 und Tab. 2 geben ein Beispiel zu diesem Faktum. Tab. 1 zeigt die Haltung gegenüber Immigranten und Immigrantinnen im ranghierarchisierten Nationenvergleich (Schweizer Bericht); Tab. 2 zeigt das Gleiche im internationalen Bericht. Die zweite Tabelle ist ohne Schärfe; es sticht eigentlich nicht in die Augen, wie das einzelne Land abschneidet. Der Leser muss schon selber allerlei Interpretationen leisten, um zu sehen, dass die Schweiz und Deutschland durchschnittlich über die Geschlechtsspezifität hinweg an unterster Stelle stehen. Diese zweite Art der Darstellung aber verstösst gegen das Prinzip des Ländervergleichs; man hebt die Olympiade auf und führt sie trotzdem durch. Man kann argumentieren, dass es 
keinen Sinn macht, so grosse Stichproben von Schülern und Schülerinnen anzuführen, wenn man im Nachhinein nicht weiss, wie sie im Vergleich zu andern Ländern politisch fühlen, wie sie handeln oder über gewisse aktuelle politische Probleme denken. Vertreter jener Nationen, die im unteren Drittel liegen, weisen denn auch darauf hin, dass man diese Daten nicht überinterpretieren dürfe, ohne die Zahl der Ausländer in einem bestimmten Land, ohne die Zahl der jährlichen Aufnahmegesuche und ohne die Zahl der jährlich Aufgenommenen im Verhältnis zur Einwohnerzahl des Landes miteinzubeziehen.

Tabelle 1: Positive Einstellungen gegenüber MigrantInnen (aus Oser et al. 2002)

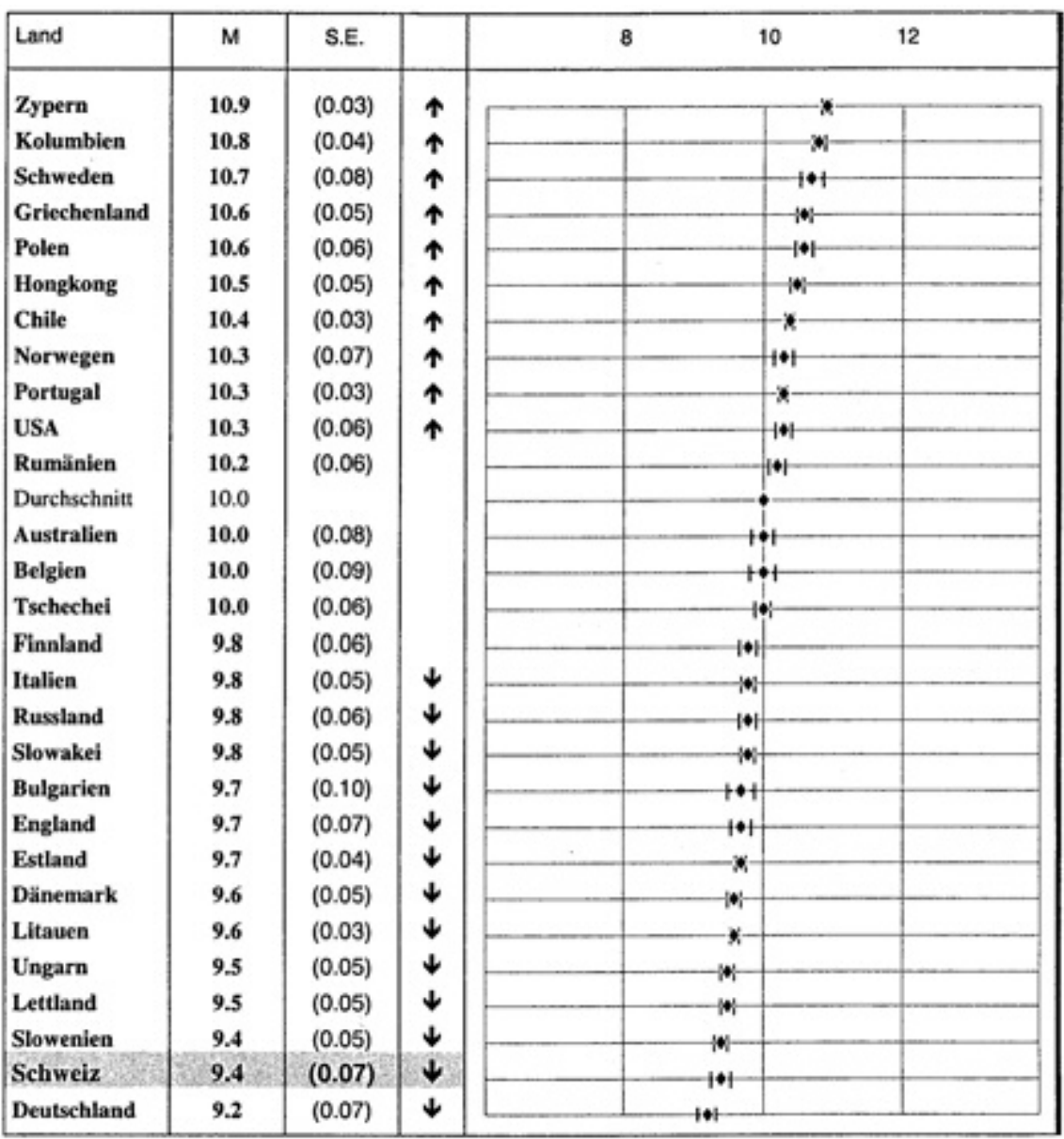

In der Tabelle ist eine Auswahl der insgesamt 28 tellnehmenden Länder dargestell. 
Tabelle 2: Positive Attitudes Toward Immigrants (aus Tourney-Purta et al. 2001, S. 105)

\begin{tabular}{|c|c|c|c|c|}
\hline Country & Mean Scale Score & 10 & 12 & \\
\hline Australia & $10.0(0.08)$ & $1 *$ & $+1+2+2$ & \\
\hline Belgium (French) & $10.0(0.09)$ & H & $H---$ & \\
\hline Bulgaria & $\boldsymbol{\nabla} \quad 9.7 \quad(0.10)$ & $1 \bullet-1$ & & \\
\hline Chile & A $10.4 \quad(0.03)$ & & 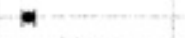 & \\
\hline Colombia & $\Delta \quad 10.8(0.04)$ & & $\cdots$ & \\
\hline Cyprus & A $10.9(0.03)$ & +4 & 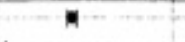 & \\
\hline Czech Republic & $10.0 \quad(0.06)$ & $-+\mid \bullet$ & & \\
\hline Denmark & $\mathbf{\nabla} 9.6(0.05)$ & $\mid \bullet$ & & \\
\hline England & $\begin{array}{lll}\mathbf{7} & 9.7 & (0.07)\end{array}$ & & & \\
\hline Estonia & $\begin{array}{r}\nabla \quad 9.7(0.04) \\
9.8(0.06)\end{array}$ & $\frac{\varphi}{\bullet}$ & & \\
\hline $\begin{array}{l}\text { Finland } \\
\text { Germany* }\end{array}$ & $\begin{array}{r}9.8(0.06) \\
\mathbf{\nabla} \quad 9.2(0.07)\end{array}$ & $\bullet \bullet$ & & \\
\hline Greece & A $10.6(0.05)$ & & $|\boldsymbol{*}|$ & \\
\hline Hong Kong (SAR) & A $10.5(0.05)$ & & 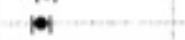 & \\
\hline Hungary & $\mathbf{\nabla} 9.5(0.05)$ & $\bullet$ & 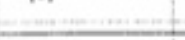 & \\
\hline Italy & $\nabla 9.8(0.05)$ & $\mid \boldsymbol{\varphi n}$ & & \\
\hline Latvia & $\boldsymbol{\nabla} \quad 9.5 \quad(0.05)$ & $|\bullet|$ & & \\
\hline Lithuania & $\boldsymbol{\nabla} \quad 9.6 \quad(0.03)$ & 叶 & & \\
\hline Norway & A $10.3(0.07)$ & & $|\bullet|$ & \\
\hline Poland & $\Delta 10.6(0.06)$ & & 10 & \\
\hline Portugal & A $10.3(0.03)$ & & 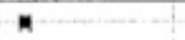 & \\
\hline Romania & $10.2 \quad(0.06)$ & & & \\
\hline Russian Federation & $9.8(0.06)$ & $+\bullet$ & & \\
\hline Slovak Republic & $\mathbf{\nabla} \quad 9.8(0.05)$ & + & & \\
\hline Sloveria & 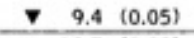 & $\bullet$ & & \\
\hline Sweden & ^ $10.7(0.08)$ & -2 & 101 & \\
\hline Switzerland* & > $9.4(0.07)$ & $+\bullet$ & & \\
\hline United States & $\Delta 10.3 \quad(0.06)$ & & $|\bullet|$ & \\
\hline \multicolumn{5}{|c|}{$\begin{array}{l}\text { () Standard errors appear in parentheses. } \\
\text { A Country mean significantly higher than intemativnal mean of } 10 \text {. }\end{array}$} \\
\hline
\end{tabular}

Source: IEA Civic Education Study, Standard Population of 14-year-olds tested in 1999.

Dies verweist auf die Frage, was eine Gesellschaft für eine politische Bildung anbieten und damit erreichen möchte. Politische Kohäsion entsteht - um ein Beispiel zu nennen - einerseits durch den Druck nationaler Bedrängnisse, die in bestimmten Zeiten eine wichtige Rolle spielen können. Sie ist typisch zu Zeiten des Krieges höher als zu Zeiten des Friedens (siehe USA nach dem 11. Sept. 2001). Kohäsion entsteht aber auch durch die Vertiefung und kritische Auseinandersetzung mit geschichtlichen Aspekten des Politischen. Was Menschen der vorhergehenden Generationen in kritischen Zeiten entschieden haben, ist keineswegs gleichgültig für die Verwurzelung in der kulturellen Tradition, dies im Negativen wie im Positiven, im Linearen wie Paradoxen (Anklagen gegenüber dem Verhalten der Schweizerbevölkerung im Zweiten Weltkrieg hat zu unerwarteten und nicht unproblematischen Kohäsionen geführt), im Konsens wie im 
Dissens. Die beiden Stimmen des Demozentrismus und des Ethnozentrismus stossen genau an solchen Positionen aufeinander; sie müssen dann je neu ausgehandelt werden, was im vorreitenden Sinne nicht zuletzt die Aufgabe der Erziehung wäre.

Wir haben an anderer Stelle dargestellt, was wir unter einer wirksamen politischen Bildung verstehen (vgl. Oser \& Reichenbach 2001; Oser \& Althof 2001), und wir haben vergleichend eine Sammlung von Ansätzen in zwei herausgegebenen Sammel- bzw. Herausgeberbänden dargestellt ${ }^{3}$. Hier sei lediglich darauf hingewiesen, dass die inhaltliche Auswahl der Skalen, die Ausprägungen im Ländervergleich, mitunter in der Tat einerseits zu Formulierungen von Erziehungszielen führen könnten, dass aber andererseits die Bildungspolitiker Desiderata formulieren müssten, die zu einer neuen geschichtlich fundierten und sprachlich artikulierbaren Balance zwischen Demozentrismus und Ethnozentrismus und ihrer entwicklungspsychologischen Dynamik führten. Politische Bildung auf der Ebene des föderalistischen Habitus kann zu einer Vereinbarung und Verpflichtung führen, die, ähnlich wie dies bei der Berufsbildung stattgefunden hat, nationale Bedeutung erhält. Wir haben genug Grundlagen; es braucht politische Entscheidungen.

Aber als «Kinder der Zufriedenheit» haben wir scheinbar keinen Anlass, beunruhigt zu sein und eine Gefahr zu wittern; wir glauben daran, dass die Bedürfnissicherung ausreicht und dass zufriedene Menschen stets irgendwie am Demokratischen hängen bleiben würden. Wir haben damit vielleicht das Politische als Bildungsziel aus den Schulen, mindestens teilweise, verbannt. In diesem Sinne ist die Bemerkung Flanagans in ihrer Studie zum Thema «The Development of Political Views and Civic Identities» (2000) von Bedeutung, nämlich dass in der Spätadoleszenz junge Menschen vordringlich nicht nur eine persönliche Identität einwickeln, sondern auch eine, die mit ihrem Verhältnis zur Gesellschaft und zum Staat zu tun hat, eine politische. Dies aber können Kantone als einzelne mindestens auf der curricularen Ebene nicht mehr alleine leisten. Die Bildungspolitiker sind deshalb dazu aufgerufen zu glauben, dass die Schule einen signifikanten Beitrag dazu leisten könnte. Modelle demokratischen Lernens durch Schulversammlungen (z. B. Just Communities) zeigen dies in Tat und gemessener Wirksamkeit. Vielleicht aber haben wir diesen Anspruch auch nur vergessen. Denn die Generationen wissen zu wenig, was vorherige Generationen geliebt haben, und der Abbau der Traditionsketten durch das Verschwinden der Geschichte aus der Gesellschaft und der Schule hat seine wahren Folgen für das Politische nur noch nicht gezeigt.

\section{Anmerkungen}

1 F. Oser, H. Biedermann, A. Haenni, C. Maiello und B. Kersten. Aus den ausgewerteten Daten entsteht ein Bericht für die Schweizer Ergebnisse (in Vorbereitung). Die finanzielle Unterstützung wurde voll umfänglich von CORECHED getragen. 
2 In einem 2001 publizierten Projekt zur Überprüfung der Lehrerbildung in der Schweiz haben wir vorausgehend kriteriell und formal festgelegt, wann wir die Qualität eines Standards als Kompetenzprofil für konkretes unterrichtliches Handeln als hoch bzw. tief bezeichnen. Dies ist ein völlig anderer Ansatz. Er geht nicht von Leistungsvergleichen der Schüler und Schülerinnen und auch nicht von internationalen Leistungsvergleichen, sondern von Expertenhandeln und -urteilen, die als Richtlinie für erfolgreiches anderes Handeln gelten, aus.

3 Reichenbach \& Oser, Hrg. "Politische Bildung und staatsbürgerliche Erziehung in der Schweiz», 1998; Reichenbach \& Oser, Hrg. "Zwischen Ernüchterung und Pathos», 2000; Oser \& Reichenbach, «Politische Bildung in der Schweiz», 2000

\section{Literaturverzeichnis}

Arendt, H. (1996). Vita Activa oder Vom tätigen Leben. München Zürich: Piper (Original: "The Human Condition» 1958).

Beck, U. (1997). Kinder der Freiheit: Wider das Lamento über den Werteverfall. In U. Beck, Kinder der Freiheit. (S. 9-33). Frankfurt: Suhrkamp.

Deutsches PISA-Konsortium (Hrsg.). (2001). PISA 2000. Basiskompetenzen von Schülerinnen und Schülern im internationalen Vergleich. Opladen: Leske+Budrich.

Flanagan, C. et. al. (2000). The Developement of Political views and Civic Identities. International Society for the Study of Behavioural Development. Newsletter, 37 (2), 4-6.

Meyer, T. (1994). Die Transformation des Politischen. Frankfurt: Suhrkamp.

Oser, F.K. \& Althoff, W. (2001). Die Gerechte Schulgemeinschaft: Lernen durch Gestaltung des Schullebens. In W. Edelstein, F. K. Oser \& P. Schuster (Hrsg.) Moralische Erziehung in der Schule. (S. 233-268). Weinheim: Beltz.

Oser, F.K., Biedermann, H., Haenni, A., Maiello, C. \& Kersten, B. (2002). Unter dem Durchschnitt. Ergebnisse der "IEA-Civic Education Studie».

Oser, F.K., Biedermann, H. \& Ullrich, M. (2001). Teilnehmen und Mitteilen: Partizipative Wege in die Res Publika. 14 Fallstudien im Rahmen des Projekts "Education à la Citoyenneté Democratique (ECD)» des Europarats. Freiburg: Departement Erziehungswissenschaften der Universität Freiburg/Schweiz.

Oser, F.K. \& Reichenbach, R. (2000). Studien und Berichte 11A. Politische Bildung in der Schweiz. Schlussbericht. Bern: Schweizerische Konferenz der kantonalen Erziehungsdirektoren.

Reichenbach, R. (1998). Abandonding the Myth of Exceptionality: On Civic Education in Switzerland. In J. Tourney-Purta, J. Schwille J.-A. Amadeo (Eds.), Civic Education Across Countries: Twenty-four National Case Studies from the IEA Civic Education Project.

Reichenach, R. (1999). Demokratisches Selbst - dilettantisches Subjekt. Zur Bedeutung demokratischer Bildung und Erziehung in der Spätmoderne. Habilitationsschrift. Freiburg/ Schweiz: Departement Erziehungswissenschaften der Universität Freiburg/Schweiz.

Amsterdam: International Association for the Evaluation of Educational Achievement.

Reichenbach, R. \& Oser, F. K. (Hrsg.). (1998). Politische Bildung und staatsbürgerliche Erziehung in der Schweiz. Perspektiven aus der Deutschschweiz und der Westschweiz. Freiburg/Schweiz: Universitätsverlag.

Reichenbach, R. \& Oser, F.K. (Hrsg.) (2000). Zwischen Pathos und Ernüchterung. Zur Lage der politischen Bildung in der Schweiz. Freiburg/Schweiz: Universitätsverlag.

Taylor, C. (2002). Wieviel Gemeinschaft braucht die Demokratie? Frankfurt: Suhrkamp.

Tourney-Purta, J., Lehmann, R., Oswal, H. \& Schulz, W. (2001). Citizenship and Education in Twenty-eight Countries. Civic Knowledge and Engagement at Age Fourteen. Amsterdam: International Association for the Evaluation of Educational Achievement. 


\section{Au dessous de la moyenne: quelques résultats, forces et faiblesses des différentes manières de présentation de comparaisons entre pays, à partir de l'exemple de l'étude IEA-Civic Education.}

\footnotetext{
Résumé

Dans cet article, deux buts sont visés. Premièrement, sera présentée une synthèse de l'étude IEA Civic Education, qui compare les données de 28 pays. Il en résulte que dans l'ensemble des domaines analysés (1. Connaissances politiques au niveau des contenus, 2. Capacités d'interprétation politique, 3. Concepts de démocratie et de citoyenneté, 4. Prises de position et 5. Actions participatives) les jeunes suisses de 14/15 ans se situent au-dessous de la moyenne internationale. Suite à ce mauvais résultat, on fait allusion au fait que les politiciens de la formation doivent réagir avec urgence. Deuxièmement, il faut tout de même se questionner sur le sens, dans le cadre de cette étude comme dans celui de PISA, de procéder avec des comparaisons entre pays autour de dimensions si différentes. Cette deuxième question sera abordée de façon critique à partir de la notion d' "olympiade de performance».
}

\section{Sotto la media: alcuni risultati, forze e debolezze delle differenti maniere di presentazione di comparazioni tra nazioni, sull'esempio dello studio IEA-Civic Education.}

\section{Riassunto}

In questo articolo sono perseguiti due scopi. Innanzi tutto, sarà presentata una sintesi dei risultati svizzeri dello studio IEA Civic Education, studio comparativo eseguito in 28 nazioni. Risulta che in tutti i campi investigati (1. Conoscenze politiche a livello di contenuti, 2. Capacità di interpretazione politica, 3. Concetti di democrazia e cittadinanza, 4. Prese di posizione e 5. Azioni partecipative) i giovani svizzeri di 14/15 anni si situano al disotto della media internazionale. Di conseguenza, viene fatta allusione al fatto che i politici della formazione debbano reagire con urgenza a questo cattivo risultato. Secondariamente, bisogna comunque chiedersi che senso abbia, in questo studio come nel quadro di PISA, procedere a dei paragoni tra paesi sulla base di dimensioni così differenti. Questa seconda domanda sarà abbordata con occhio critico dal punto di vista della nozione di «olimpiade di prestazione». 


\section{Below the average: Some results, strengths and weaknesses of differrent way of representing comparisons by country, exemplified by the IEA Civic Education study.}

\section{Abstract}

This paper is aimed at the two following goals: First, an overview of the Swiss results of the IEA study on Civic Education, a comparative study across 28 countries, is presented. Regarding most major topics (civic knowledge; civic skills; civic concepts with respect to democracy and citizenship; civic attitudes, and participatory action) the Swiss students aged $14 / 15$ years score below the mean, as compared to the 27 other countries. On the one hand, these results put politicians in the field of education under the pressure to change something. On the other hand, we are urged to raise, as the second goal, the question whether this type of international comparison studies (PISA as another example) is somehow politically incorrect or obsolete. This second question is treated under the heading «Intellectual Olympic Games». 
T h e m a 